\title{
A NEW APPROACH TO THE CORONA THEOREM FOR DOMAINS BOUNDED BY A $C^{1+\alpha}$ CURVE
}

\author{
José Manuel Enríquez-Salamanca and María José González \\ University of Cádiz, Department of Mathematics \\ Cádiz, 11002, Spain; enrique.desalamanca@uca.es \\ University of Cádiz, Department of Mathematics \\ Puerto Real, 11510, Spain; majose.gonzalez@uca.es
}

\begin{abstract}
We prove the corona theorem for domains whose boundary lies in a $C^{1+\alpha}$ curve. For that, we transfer $H^{\infty}$ on the complement of the curve onto a Denjoy domain and use the results from Garnett and Jones.
\end{abstract}

\section{Introduction}

Let $\Gamma$ be an unbounded $C^{1+\alpha}$ curve analytic at $\infty, E$ a compact subset of this curve with positive length and set $\Omega=\mathbf{C}^{*} \backslash E$. Let us denote the space of bounded analytic functions on $\Omega$ by $H^{\infty}(\Omega)$. The corona theorem for this type of domains was already proved by Moore in [7]. The purpose of this paper is to present a new approach to this result.

Theorem 1. Let $f_{1}, f_{2}, \ldots, f_{n} \in H^{\infty}(\Omega)$ so that $\delta \leq \max _{k}\left|f_{k}(\omega)\right| \leq 1$, for all $\omega \in \Omega$ and some $\delta>0$. Then, there exist $g_{1}, g_{2}, \ldots, g_{n} \in H^{\infty}(\Omega)$ such that $f_{1} g_{1}+f_{2} g_{2}+\ldots+f_{n} g_{n}=1$ on $\Omega$.

The functions $\left\{f_{k}\right\}_{k=1}^{n}$ and $\left\{g_{k}\right\}_{k=1}^{n}$ are called corona data and corona solutions respectively, and $\delta$ and $n$ are the corona constants. When $\Gamma$ is the real line, the domain $\Omega$ is called a Denjoy domain. In this case, the theorem was proved by Garnett and Jones [5].

The first corona problem for simply connected domains was solved by Carleson in 1962 [1]. Since then, the result has been extended to some classes of infinitely connected domains, in particular to domains whose boundary lies in a Lipschitz graph and satisfies a thickness condition [8] or complements of Cantor sets [6].

For our approach, we will apply the following result proved in [2] which allows us to transfer the problem in $\Omega$ to a Denjoy domain.

Theorem 2. Let $\Gamma$ be an unbounded $C^{1+\alpha}$ curve analytic at $\infty$, and let $\rho$ denote a conformal map of $\mathbf{R}_{-}^{2}$ onto any of the regions bounded by $\Gamma$. Then, given a function $g \in L^{\infty}(\Gamma)$, the Cauchy integral $C_{\Gamma}(g) \in L^{\infty}(\mathbf{C})$ if and only if $C_{\mathbf{R}}(f) \in L^{\infty}(\mathbf{C})$, where $f$ denotes the pullback of $g$ under the conformal mapping $\rho$.

This transfer is possible thanks to the existence of a quasiconformal extension of $\rho$ whose complex dilatation, $\mu$, verifies that $|\mu|^{2} /|y|^{1+\varepsilon} d x d y$ is a Carleson measure

doi:10.5186/aasfm.2015.4043

2010 Mathematics Subject Classification: Primary 30H80.

Key words: Cauchy integral, quasiconformal mapping, Carleson measure, smooth curve, corona theorem.

The second author is supported by Grant MTM 2011-24606. 
relative to $\mathbf{R}$ for some $\varepsilon=\varepsilon(\alpha)>0$. In fact, the existence of such an extension characterizes $C^{1+\alpha}$ curves [2, Theorem 1].

The paper is structured as follows: In section 1, we review some definitions and basic facts. The proof of Theorem 1 is presented in section 2 .

\section{Preliminaries}

Let us denote complex variables by $z=x+i y$ and $\omega=\xi+i \eta . B_{r}(z)$ will denote the ball centered at $z$ and radius $r$ and $C$ will represent a positive constant that could be different throughout an inequality. Also, we shall write $\bar{\partial}=\partial / \partial \bar{z}=1 / 2\left(\partial_{x}+i \partial_{y}\right)$ and $\partial=\partial / \partial z=1 / 2\left(\partial_{x}-i \partial_{y}\right)$. For a square $Q$, we will denote by $\alpha Q, \alpha>0$, the dilation of this square by a scale factor $\alpha$ and by $l(Q)$ its length.

A Jordan curve $\Gamma$ is said to be of class $C^{n}(n=1,2, \ldots)$ if it has a parametrization $\varphi(\tau)=f\left(e^{i \tau}\right), 0 \leq \tau \leq 2 \pi$, that is $n$ times continuously differentiable and satisfies that $\varphi^{\prime}(\tau) \neq 0, \forall \tau$. Furthermore, it is of class $C^{n+\alpha}$, for $0<\alpha<1$, if

$$
\left|\varphi^{(n)}\left(\tau_{1}\right)-\varphi^{(n)}\left(\tau_{2}\right)\right| \leq C\left|\tau_{1}-\tau_{2}\right|^{\alpha} .
$$

Given a function $F$ on $\Gamma$ define its Cauchy integral $f(z)=C_{\Gamma}(F)(z)$ off $\Gamma$ by

$$
f(z)=\frac{1}{2 \pi \mathrm{i}} \int_{\Gamma} \frac{F(\zeta)}{\zeta-z} d \zeta, \quad \zeta \notin \Gamma .
$$

We define the jump of $f=C_{\Gamma}(F)$ across $\Gamma$ at a point $z, \mathrm{j}(f)(z)$, as $f_{+}(z)-f_{-}(z)$, where $f_{+}$and $f_{-}$denote the boundary values of $f$. As the classical Plemelj formula states,

$$
f_{ \pm}(z)= \pm \frac{1}{2} F(z)+\frac{1}{2 \pi i} P . V . \int_{\Gamma} \frac{F(\omega)}{\omega-z} d \omega, \quad z \in \Gamma .
$$

Hence $f_{+}(z)-f_{-}(z)=F(z)$. Also, $f$ is holomorphic off $\Gamma$ so that $\bar{\partial} f=0$ on $\mathbf{C} \backslash \Gamma$.

A positive measure $\lambda$ on $\mathbf{C}$ is called a Carleson measure relative to a given chordarc curve $\Gamma$ if there exists a constant $C>0$ such that $\lambda\left(B_{R}(z)\right) \leq C R$ for all $z \in \Gamma$ and $R>0$. The smallest such $C$ is the norm of $\lambda,\|\lambda\|_{C}$. Furthermore, if

$$
\limsup _{r \rightarrow 0} \frac{\lambda\left(B_{z}(R)\right)}{R}=0,
$$

then we say that $\lambda$ is a vanishing Carleson measure or that it satisfies a o(1)-Carleson condition.

\section{Proof of the Theorem}

Let $\Omega_{+}$and $\Omega_{-}$be the two regions bounded by the $C^{1+\alpha}$ curve $\Gamma$ and $\rho$ be a conformal map from $\mathbf{R}_{-}^{2}$ onto $\Omega_{-}$. It was proved in [2] that $\rho$ extends to a global quasiconformal map whose dilatation $\mu$ satisfies that $\nu=|\mu|^{2} /|y|^{1+\varepsilon} d x d y$ is a Carleson measure relative to $\mathbf{R}$ where $\varepsilon=\varepsilon(\alpha)$. In fact, for this extension, it holds that $|\partial \rho(z)| \simeq\left|\rho^{\prime}(\bar{z})\right|$ if $0<\operatorname{Im}(z)<\varepsilon_{0}$ for some $\varepsilon_{0}=\varepsilon_{0}(\alpha)$ small enough [2, Proof of Theorem 1].

Besides, since $\Gamma$ is analytic at $\infty$, we will assume that $\mu$ has compact support. We will keep the notation fixed for the rest of the proof, that is, $\rho$ is a quasiconformal mapping associated to $\Gamma, \mu$ is its complex dilatation and $\varepsilon$ is such that $\nu$ is a Carleson measure. 
Let $E_{0}=\rho^{-1}(E) \subset \mathbf{R}$ and $\Omega_{0}=\mathbf{C} \backslash E_{0}$. Note that $E_{0}$ is closed and has positive length ([9], Theorem 6.8). Define the space

$$
H^{\infty}\left(\Omega_{0}, \mu\right)=\left\{f \circ \rho: f \in H^{\infty}(\Omega)\right\} .
$$

Observe that if $g=f \circ \rho \in H^{\infty}\left(\Omega_{0}, \mu\right)$, then $\bar{\partial} f=0$ on $\Omega$ translates into $(\bar{\partial}-\mu \partial) g=0$ on $\Omega_{0}$, and as well, the jump of $g$ across $E_{0}$ is given by $\mathrm{j}(g)=\mathrm{j}(f) \circ \rho$. Also, as $\Gamma$ is a $C^{1+\alpha}$ curve, $\lambda=|y||\partial g|^{2} d x d y$ is a Carleson measure relative to $\mathbf{R}[2$, Proof of Theorem 2].

Before proving the corona theorem, we need some preliminary lemmas.

Lemma 2.1. If $g \in H^{\infty}\left(\Omega_{0}, \mu\right)$, then $\tau=|\mu||\partial g| d x d y$ is a vanishing Carleson measure.

Proof. For any $s \in \mathbf{R}, r>0$ :

$$
\int_{B_{r}(s)} \frac{|\mu(z)|^{2}}{|y|} d x d y=\int_{B_{r}(s)} \frac{|\mu(z)|^{2}}{|y|^{1+\varepsilon}}|y|^{\varepsilon} d x d y \lesssim\|\nu\|_{C} r^{1+\varepsilon} .
$$

Therefore,

$$
\begin{aligned}
\int_{B_{r}(s)}|\mu(z) \partial g(z)| d x d y & \leq\left(\int_{B_{r}(s)} \frac{|\mu(z)|^{2}}{|y|} d x d y\right)^{1 / 2}\left(\int_{B_{r}(s)}|\partial g(z)|^{2}|y| d x d y\right)^{1 / 2} \\
& \lesssim\|\nu\|_{C}^{1 / 2}\|\lambda\|_{C}^{1 / 2} r^{1+\varepsilon / 2}
\end{aligned}
$$

and $\tau=|\mu||\partial g| d x d y$ is a vanishing Carleson measure relative to $\mathbf{R}$.

Lemma 2.2. There exists $\varepsilon_{0}>0$ such that if $g \in H^{\infty}\left(\Omega_{0}, \mu\right)$ and $z \in \Omega_{0}$ with $0<|\operatorname{Im}(\mathrm{z})|<\varepsilon_{0}$, then $|y||\partial g(z)|<C$, where $C=C\left(\|g\|_{\infty},\|\mu\|_{\infty}\right)$ and $\varepsilon_{0}=\varepsilon_{0}(\alpha)$.

Proof. Let $f \in H^{\infty}(\Omega)$ such that $g=f \circ \rho$. Then, $\delta_{\Gamma}(\omega)\left|f^{\prime}(\omega)\right| \leq C, \forall \omega \in \mathbf{C} \backslash \Gamma$ and $C=C\left(\|f\|_{\infty}\right)$.

Let $z \in \mathbf{R}_{-}^{2}$ and $\omega=\rho(z)$. Since $\rho$ is conformal on $\mathbf{R}_{-}^{2}$, by Koebe's distortion theorem,

$$
|y||\partial g(z)|=|y|\left|f^{\prime}(\rho(z))\right|\left|\rho^{\prime}(z)\right| \simeq \delta_{\Gamma}(\omega)\left|f^{\prime}(\omega)\right| \leq C .
$$

If $z \in \mathbf{R}_{+}^{2}$, as we mentioned before, we can choose $\varepsilon_{0}$ so that, if $0<|\operatorname{Im}(z)|<\varepsilon_{0}$ then, $|\partial \rho(z)| \simeq\left|\rho^{\prime}(\bar{z})\right|$. Hence, as above

$$
|y||\partial g(z)|=|y||\partial \rho(z)|\left|f^{\prime}(\rho(z))\right| \simeq \delta_{\Gamma}(\rho(\bar{z}))\left|f^{\prime}(\rho(z))\right| .
$$

By the distortion theorem for quasiconformal mappings $\delta_{\Gamma}(\rho(\bar{z})) \simeq \delta_{\Gamma}(\rho(z))$ with comparison constants depending on $\|\mu\|_{\infty}$, which concludes the proof.

Before stating the next lemma, we will review some facts already developed in [2] which follow Semmes's approach in [10]. Let $g \in H^{\infty}\left(\Omega_{0}, \mu\right)$, then $g=f \circ \rho$ for some $f \in H^{\infty}(\Omega)$. Consider now the jump of $g, \mathrm{j}(g)$, and set $\tilde{g}=C_{\mathbf{R}}(\mathrm{j}(g))$. If we define $G=g-\tilde{g}$, then $\bar{\partial} G=\mu \partial g$ on $\Omega_{0}$ and since $G$ has no jump across $E_{0}$, we can consider that this equation holds on all $\mathbf{C}$ in the sense of distributions. We can then apply Cauchy's formula to obtain

$$
G\left(z_{0}\right)=\frac{1}{\pi \mathrm{i}} \int_{\mathbf{C}} \frac{\bar{\partial} G(z)}{z-z_{0}} d x d y=\frac{1}{\pi \mathrm{i}} \int_{\mathbf{C}} \frac{\mu(z) \partial g(z)}{z-z_{0}} d x d y, \quad \text { for all } z_{0} \in \mathbf{C} .
$$

Lemma 2.3. Assume that $\operatorname{supp}(\mu) \subset Q$ for some $Q$ centered at a real point with length $R \leq \varepsilon_{0} / 4$. Let $g \in H^{\infty}\left(\Omega_{0}, \mu\right)$ and $\tilde{g} \in H^{\infty}\left(\Omega_{0}\right)$ so that $\mathrm{j}(g)=\mathrm{j}(\tilde{g})$ and set $G=g-\tilde{g}$. Then for all $z \in \mathbf{C},|G(z)| \leq C R^{\varepsilon /(2+\varepsilon)}$, where $C=C\left(\|g\|_{\infty},\|\nu\|_{C}\right)$. 

then

Proof. Consider $z_{0}=x_{0}+\mathrm{i} y_{0} \in(2 Q \backslash \mathbf{R})$. Since $\bar{\partial} G=\mu \partial g$ and $\operatorname{supp}(\mu) \subset Q$,

$$
\begin{aligned}
\left|G\left(z_{0}\right)\right| & \lesssim \int_{\mathbf{C}} \frac{|\mu(z) \partial g(z)|}{\left|z-z_{0}\right|} d x d y=\int_{Q} \frac{|\mu(z) \partial g(z)|}{\left|z-z_{0}\right|} d x d y \\
& =\int_{Q_{0}} \frac{|\mu(z) \partial g(z)|}{\left|z-z_{0}\right|} d x d y+\int_{Q \backslash Q_{0}} \frac{|\mu(z) \partial g(z)|}{\left|z-z_{0}\right|} d x d y
\end{aligned}
$$

where $Q_{0}$ is the square centered at $z_{0}$ and length $l\left(Q_{0}\right)=\left|y_{0}\right|$. To bound the first integral in (2), set $p=2+\varepsilon$ and $q=(2+\varepsilon) /(1+\varepsilon)$. Then

(3) $\int_{Q_{0}} \frac{|\mu(z) \partial g(z)|}{\left|z-z_{0}\right|} d x d y \leq\left(\int_{Q_{0}}|\mu(z) \partial g(z)|^{2+\varepsilon} d x d y\right)^{\frac{1}{2+\varepsilon}}\left(\int_{Q_{0}}\left|z-z_{0}\right|^{-\frac{2+\varepsilon}{1+\varepsilon}} d x d y\right)^{\frac{1+\varepsilon}{2+\varepsilon}}$.

As $\nu=|\mu|^{2} /|y|^{1+\varepsilon}$ is a Carleson measure relative to $\mathbf{R}$ and $|y| \geq\left|y_{0}\right| / 2$ for $z \in Q_{0}$, we obtain by lemma 2.2 :

$$
\begin{aligned}
\int_{Q_{0}}|\mu(z) \partial g(z)|^{2+\varepsilon} d x d y & \lesssim \int_{Q_{0}}|\mu(z)|^{2+\varepsilon} \frac{1}{|y|^{2+\varepsilon}} d x d y \\
& \lesssim \frac{2}{\left|y_{0}\right|} \int_{2 Q_{0}} \frac{|\mu(z)|^{2}}{|y|^{1+\varepsilon}} d x d y \leq 4\|\nu\|_{C} .
\end{aligned}
$$

Let us now consider $B_{0}=B_{r}\left(z_{0}\right)$ so that $r \simeq\left|y_{0}\right|$ and $Q_{0} \subset B_{0}$. By changing variables to polar coordinates,

$$
\int_{Q_{0}}\left|z-z_{0}\right|^{-\frac{2+\varepsilon}{1+\varepsilon}} d x d y \leq \int_{B_{0}}\left|z-z_{0}\right|^{-\frac{2+\varepsilon}{1+\varepsilon}} d x d y \leq C(\varepsilon) r^{\frac{\varepsilon}{1+\varepsilon}} \simeq C(\varepsilon)\left|y_{0}\right|^{\frac{\varepsilon}{1+\varepsilon}} .
$$

Therefore, by (3), (4) and (5)

$$
\int_{Q_{0}} \frac{|\mu(z) \partial g(z)|}{\left|z-z_{0}\right|} d x d y \lesssim C\left(\|\nu\|_{C}, \varepsilon\right)\left|y_{0}\right|^{\frac{\varepsilon}{2+\varepsilon}} \lesssim C\left(\|\nu\|_{C}, \varepsilon\right) R^{\frac{\varepsilon}{2+\varepsilon}} .
$$

To bound the second integral in (2), consider an open cover of $Q \backslash Q_{0}$ with squares, $Q_{i}$, centered at $z_{0}$ and length $l\left(Q_{i}\right)=2^{i}\left|y_{0}\right|, i \geq 1$. Note that it is sufficient a cover with $M$ squares such that $M \lesssim \log _{2}\left(R /\left|y_{0}\right|\right)$. Then, by (1)

$$
\begin{aligned}
\int_{Q \backslash Q_{0}} \frac{|\mu(z) \partial g(z)|}{\left|z-z_{0}\right|} d x d y & \lesssim \sum_{i=1}^{M} \frac{2^{-i}}{\left|y_{0}\right|} \int_{Q_{i} \backslash Q_{i-1}}|\mu(z) \partial g(z)| d x d y \\
& \lesssim \sum_{i=1}^{M} \frac{2^{-i}}{\left|y_{0}\right|}\left(2^{i}\left|y_{0}\right|\right)^{1+\varepsilon / 2} \lesssim\left|y_{0}\right|^{\varepsilon / 2}\left(2^{\varepsilon / 2}\right)^{M} \lesssim R^{\varepsilon / 2}
\end{aligned}
$$

Therefore, by (2), (6) and (7), $\left|G\left(z_{0}\right)\right| \leq C\left(\|\nu\|_{C},\|\lambda\|_{C}, \varepsilon\right) R^{\varepsilon /(2+\varepsilon)}$.

For $z_{0} \in(2 Q \bigcap \mathbf{R})$, let $Q^{i}$ be the square centered at $z_{0}$ and length $l\left(Q^{i}\right)=2^{2-i} R$, $i \geq 0$. Since $\bar{\partial} G=\mu \partial g$ and $\operatorname{supp}(\mu) \subset Q$, by (1)

$$
\begin{aligned}
\left|G\left(z_{0}\right)\right| & \lesssim \int_{Q} \frac{|\mu(z) \partial g(z)|}{\left|z-z_{0}\right|} d x d y=\sum_{i \geq 0} \int_{Q^{i} \backslash Q^{i+1}} \frac{|\mu(z) \partial g(z)|}{\left|z-z_{0}\right|} d x d y \\
& \lesssim \frac{1}{R} \sum_{i \geq 0} 2^{i} \int_{Q^{i}}|\mu(z) \partial g(z)| d x d y \lesssim \frac{1}{R} \sum_{i \geq 0} 2^{i} l\left(Q^{i}\right)^{1+\varepsilon / 2} \lesssim R^{\varepsilon / 2} .
\end{aligned}
$$

Therefore, $\left|G\left(z_{0}\right)\right| \leq C R^{\varepsilon} / 2$ for $C=C\left(\|\mu\|_{C},\|\lambda\|_{C}, \varepsilon\right)$. 
Finally, let $z_{0} \in \mathbf{C} \backslash 2 Q$. Then, by (1)

$$
\begin{aligned}
\left|G\left(z_{0}\right)\right| & \lesssim \int_{Q} \frac{|\mu(z) \partial g(z)|}{\left|z-z_{0}\right|} d x d y \leq \frac{1}{R} \int_{Q}|\mu(z) \partial g(z)| d x d y \\
& \leq C\left(\|\nu\|_{C},\|\lambda\|_{C}\right) R^{\varepsilon / 2} .
\end{aligned}
$$

We now prove Theorem 1:

Theorem 1. Let $f_{1}, f_{2}, \ldots, f_{n} \in H^{\infty}(\Omega)$ so that $\delta \leq \max _{j}\left|f_{j}(\omega)\right| \leq 1$, for all $\omega \in \Omega$ and some $\delta>0$. Then, there exist $g_{1}, g_{2}, \ldots, g_{n} \in H^{\infty}(\Omega)$ such that $f_{1} g_{1}+f_{2} g_{2}+\ldots+f_{n} g_{n}=1$ on $\Omega$.

Proof. Gamelin [3] showed that it is sufficient to prove it locally, that is, that for $\zeta \in \Gamma$ there exists a neighborhood of $\zeta$ on which it is true and such that the size of this neighborhood is determined by $\delta, n$ and other parameters concerning $\Gamma$ (see also [4, p. 358]).

We can then assume that $\mu(z)=0$ outside a square $Q$ centered at a real point with length $R$, for a small enough $R=R(n, \delta, \Gamma)$ to be determined later. To see this, consider the solution $\tilde{\rho}$ of the Beltrami equation $\bar{\partial} \tilde{\rho}=\mu \partial \tilde{\rho}$ for $z \in Q, \bar{\partial} \tilde{\rho}=0$ otherwise. Then, $\rho=F \circ \tilde{\rho}$ where $F$ is an univalent function in the region $\tilde{\rho}(Q)$, and therefore it will be enough to prove the corona theorem for the domain $\tilde{\Omega}=\mathbf{C} \backslash \tilde{\rho}\left(E_{0}\right)$.

Since the dilatation coefficient $\tilde{\mu}=\mu \chi_{Q}$ obviously satisfies that $|\tilde{\mu}|^{2} /|y|^{1+\varepsilon} d x d y$ is a Carleson measure, we know that $\tilde{\Gamma}=\tilde{\rho}(\mathbf{R})$ is also a $C^{1+\tilde{\alpha}}$ curve for $\tilde{\alpha}=\tilde{\alpha}\left(\alpha,\|\mu\|_{\infty}\right)$ ([2], Theorem 1) and therefore all the previous lemmas apply if we replace $\Gamma, \mu$ and $\rho$ by the corresponding $\tilde{\Gamma}, \tilde{\mu}$ and $\tilde{\rho}$. To avoid excessive use of notation, we will drop the tilde notation.

Let $f_{k}^{*}=f_{k} \circ \rho$ on $\Omega_{0}$. Then, the jump of $f_{k}^{*}$ across $E_{0}$ is indeed the pullback of $\mathrm{j}\left(f_{k}\right)$ under the mapping $\rho$, that is, $\mathrm{j}\left(f_{k}^{*}\right)=\mathrm{j}\left(f_{k}\right) \circ \rho$. Note that $f_{1}^{*}, \ldots, f_{n}^{*} \in$ $H^{\infty}\left(\Omega_{0}, \mu\right)$.

Set $\tilde{f}_{k}=C_{\mathbf{R}}\left(\mathrm{j}\left(f_{k}^{*}\right)\right)$. By Theorem $2, \tilde{f}_{k} \in H^{\infty}\left(\Omega_{0}\right)$. First, we want to show that $\tilde{f}_{k}$ are corona data in $\Omega_{0}$. So, let $G_{k}=f_{k}^{*}-\tilde{f}_{k}$ and $z_{0} \in \Omega_{0}$. Then, there exists $1 \leq j \leq n$ such that $\delta \leq\left|f_{j}^{*}\left(z_{0}\right)\right| \leq\left|G_{j}\left(z_{0}\right)\right|+\left|\tilde{f}_{j}\left(z_{0}\right)\right|$. By lemma $2.3,\left|G_{j}\left(z_{0}\right)\right| \lesssim R^{\varepsilon /(2+\varepsilon)} \leq \delta / 2$ for a sufficiently small $R$ and therefore $\delta / 2 \leq\left|\tilde{f}_{j}\left(z_{0}\right)\right|$.

According to Garnett and Jones' theorem for Denjoy domains [5], there exist $\tilde{h}_{1}, \tilde{h}_{2}, \ldots, \tilde{h}_{n} \in H^{\infty}\left(\Omega_{0}\right)$ such that $\tilde{f}_{1} \tilde{h}_{1}+\ldots \tilde{f}_{n} \tilde{h}_{n}=1$ with $\left\|\tilde{h}_{k}\right\|_{\infty} \leq C(n, \delta)$.

Define $H_{k}^{*}=\mathrm{j}\left(\tilde{h}_{k}\right)$. Then, $H_{k}^{*} \in L^{\infty}(\mathbf{R})$ and $\tilde{h}_{k}=C_{\mathbf{R}}\left(H_{k}^{*}\right)$. Set $H_{k}=H_{k}^{*} \circ \rho^{-1}$ on $\Gamma$ and define $h_{k}=C_{\Gamma}\left(H_{k}\right)$. Although $\left\{h_{k}\right\}_{k=1}^{n} \subset H^{\infty}(\Omega)$ by Theorem 2, they are not corona solutions as they do not verify that $f_{1} h_{1}+f_{2} h_{2}+\ldots f_{n} h_{n}=1$ on $\Omega$.

Consider the analytic functions $g_{k}(\omega)=h_{k}(\omega) /\left(\sum f_{j}(\omega) h_{j}(\omega)\right), 1 \leq k \leq n$, on $\Omega$. They clearly satisfy that $\sum g_{j} f_{j}=1$. We just need to prove that $g_{1}, g_{2}, \ldots g_{n}$ are also bounded. For that, it is sufficient to show that $\sum f_{k} h_{k}$ is close to 1 .

Let us denote $h_{k}^{*}=h_{k} \circ \rho \in H^{\infty}\left(\Omega_{0}, \mu\right)$. Note that $\mathrm{j}\left(h_{k}^{*}\right)=\mathrm{j}\left(\tilde{h}_{k}\right)$. For any $z \in \Omega_{0}$ and by lemma 2.3:

$$
\begin{aligned}
& \left|\sum_{k=1}^{n} f_{k}(\rho(z)) h_{k}(\rho(z))-1\right|=\left|\sum_{k=1}^{n} f_{k}^{*}(z) h_{k}^{*}(z)-\sum_{i=1}^{n} \tilde{f}_{k}(z) \tilde{h}_{k}(z)\right| \\
& \leq \sum_{k=1}^{n}\left|f_{k}^{*}(z)\right|\left|h_{k}^{*}(z)-\tilde{h}_{k}(z)\right|+\sum_{k=1}^{n}\left|\tilde{h}_{k}(z)\right|\left|\tilde{f}_{k}(z)-f_{k}^{*}(z)\right|
\end{aligned}
$$




$$
\lesssim n R^{\varepsilon /(2+\varepsilon)}+n C(n, \delta) R^{\varepsilon /(2+\varepsilon)} \leq 1 / 2
$$

for a sufficiently small $R$.

As a final remark, this new approach encourages us to find solutions to the corona problem for domains bounded by other quasicircles. For that, one would need to find conditions on $\mu$ so that we can transfer $H^{\infty}$ on the complement of a curve onto the corresponding Denjoy domain.

\section{References}

[1] Carleson, L.: Interpolations by bounded analytic functions on the corona theorem. - Ann. of Math. (2) 76, 1962, 547-559.

[2] Enríquez-Salamanca, J. M., and M. J. GonzÁlez: On $H^{\infty}$ on the complement of $C^{1+\alpha}$ curves. - J. Math. Anal. Appl. 416:1, 2014, 335-343.

[3] Gamelin, T. W.: Localization of the corona problem. - Pacific J. Math. 34, 1970, 73-81.

[4] Garnett, J. B.: Bounded analytic functions. - Academic Press, New York, 1981.

[5] Garnett, J. B., and P. W. Jones: The corona theorem for Denjoy domains. - Acta Math. $155,1985,27-40$.

[6] Handy, J.: The corona theorem on the complement of certain square Cantor sets. - J. Anal. Math. 108, 2009, 1-18.

[7] Moore, C. N.: The corona Theorem for domains whose boundary lies in a smooth curve. Proc. Amer. Math. Soc. 100, 1987, 266-270.

[8] Newdelman, B. M.: Homogeneous subsets of a lipschitz graph and the corona theorem. Publ. Mat. 55, 2011, 93-121.

[9] Pommerenke, Ch.: Boundary behaviour of conformal maps. - Springer-Verlag, Berlin, 1973.

[10] Semmes, S.: Estimates for $(\bar{\partial}-\nu \partial)^{-1}$ and Calderon's theorem on the Cauchy integral. - Trans. Amer. Math. Soc. 306, 1988, 191-232. 